\title{
Supporting Information: Stretched Poly(acrylonitrile) as a Scalable Alignment Medium for DMSO
}

\author{
Grit Kummerlöwe, ${ }^{\dagger}$ Jörg Auernheimer, ${ }^{\dagger, \ddagger}$ Andreas Lendlein, ${ }^{\S}$ Burkhard Luy ${ }^{*, \dagger}$ \\ ${ }^{\dagger}$ Lehrstuhl für Organische Chemie II, Department Chemie, Technische Universität München, \\ Lichtenbergstraße 4, 85747 Garching, Germany. ${ }^{\ddagger}$ Klinikum rechts der Isar der Technischen Universität München, \\ Nuklearmedizinische Klinik und Poliklinik, Ismaningerstr. 22, 81675 München, Germany. ${ }^{\S}$ Center for Biomaterial \\ Development, Institute of Polymer Research GKSS Research Center, Kantstr. 55, 14513 Teltow, Germany.
}

RECEIVED DATE (automatically inserted by publisher); E-mail: burkhard.luy@ch.tum.de
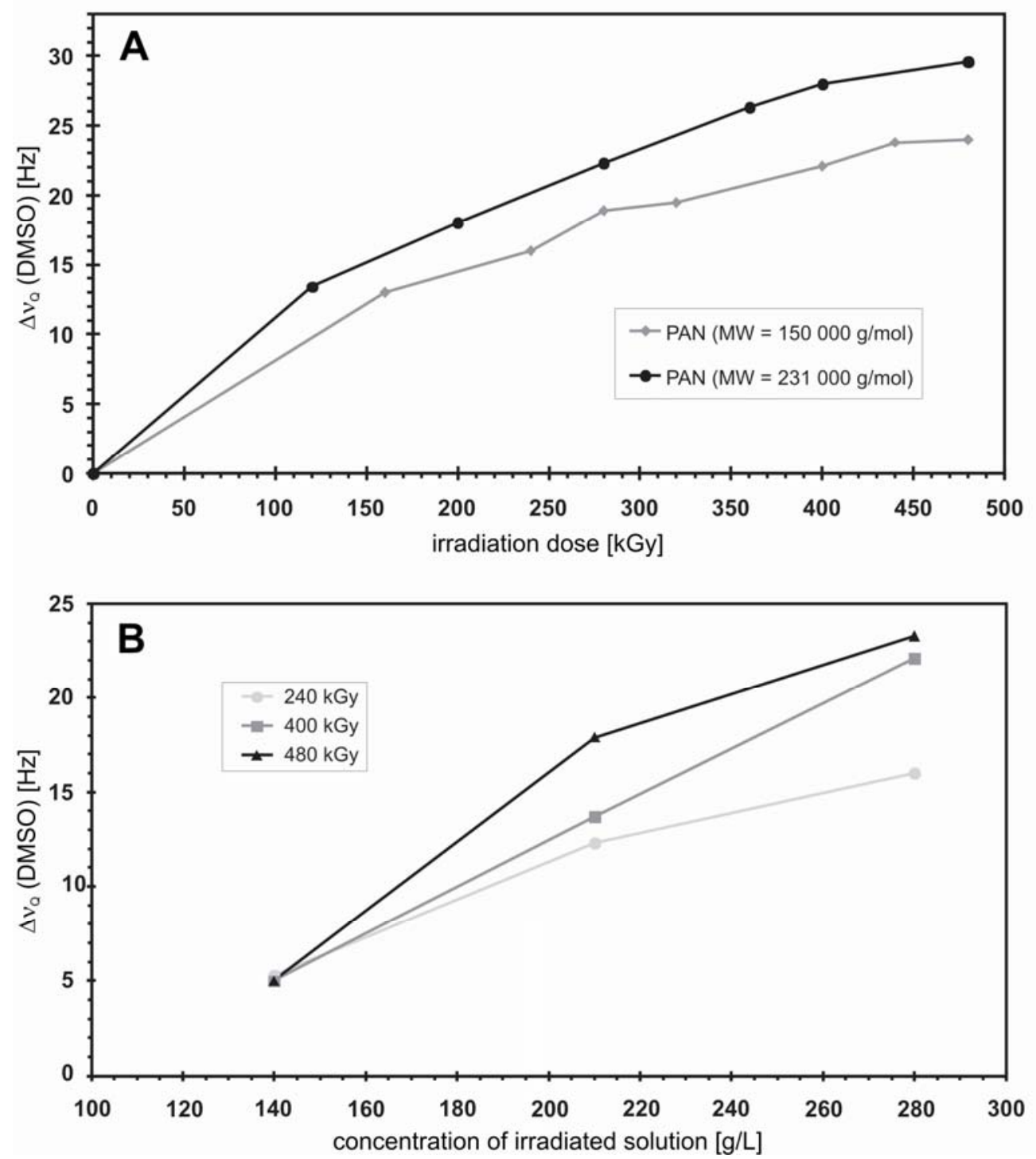

Figure S1. Scalability of alignment. (A) Quadrupolar splitting $\Delta v_{\mathrm{Q}}$ of PAN/DMSO-gels with respect to the irradiation dose applied for cross-linking. All gels were cross-linked inside the NMR-tube as a $280 \mathrm{~g} / \mathrm{L}$ PAN/DMSO- $\mathrm{d}_{6}$ solution. Linear polymers of two different molecular weights have been used. (B) Quadrupolar splitting $\Delta v_{\mathrm{Q}}$ of various gels with respect to the initial concentration of the PAN (MW=150000 $\mathrm{g} / \mathrm{mol}$ )/DMSO- $\mathrm{d}_{6}$ solution used for irradiation with accelerated electrons. All samples were measured after 57 days of equilibration in silylated NMR-tubes. 


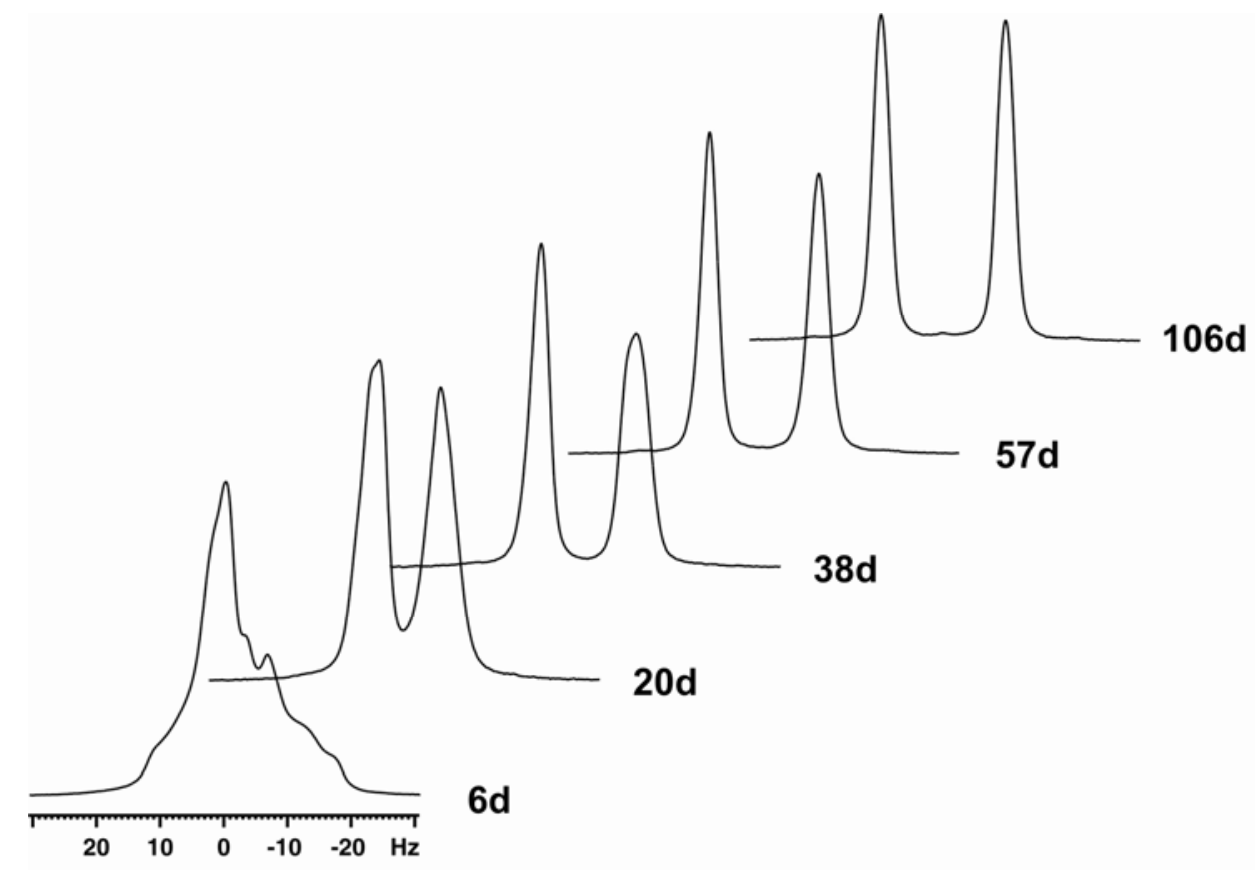

Figure S2. Equilibration of a PAN/DMSO-gel at room temperature in a silylated NMR-tube (irradiation dose = $320 \mathrm{kGy}$; final quadrupolar splitting $\Delta v_{\mathrm{Q}}=19.6 \mathrm{~Hz}$ ) monitored by the ${ }^{2} \mathrm{H}-1 \mathrm{D}$ spectrum of DMSO-d $\mathrm{d}_{6}$.

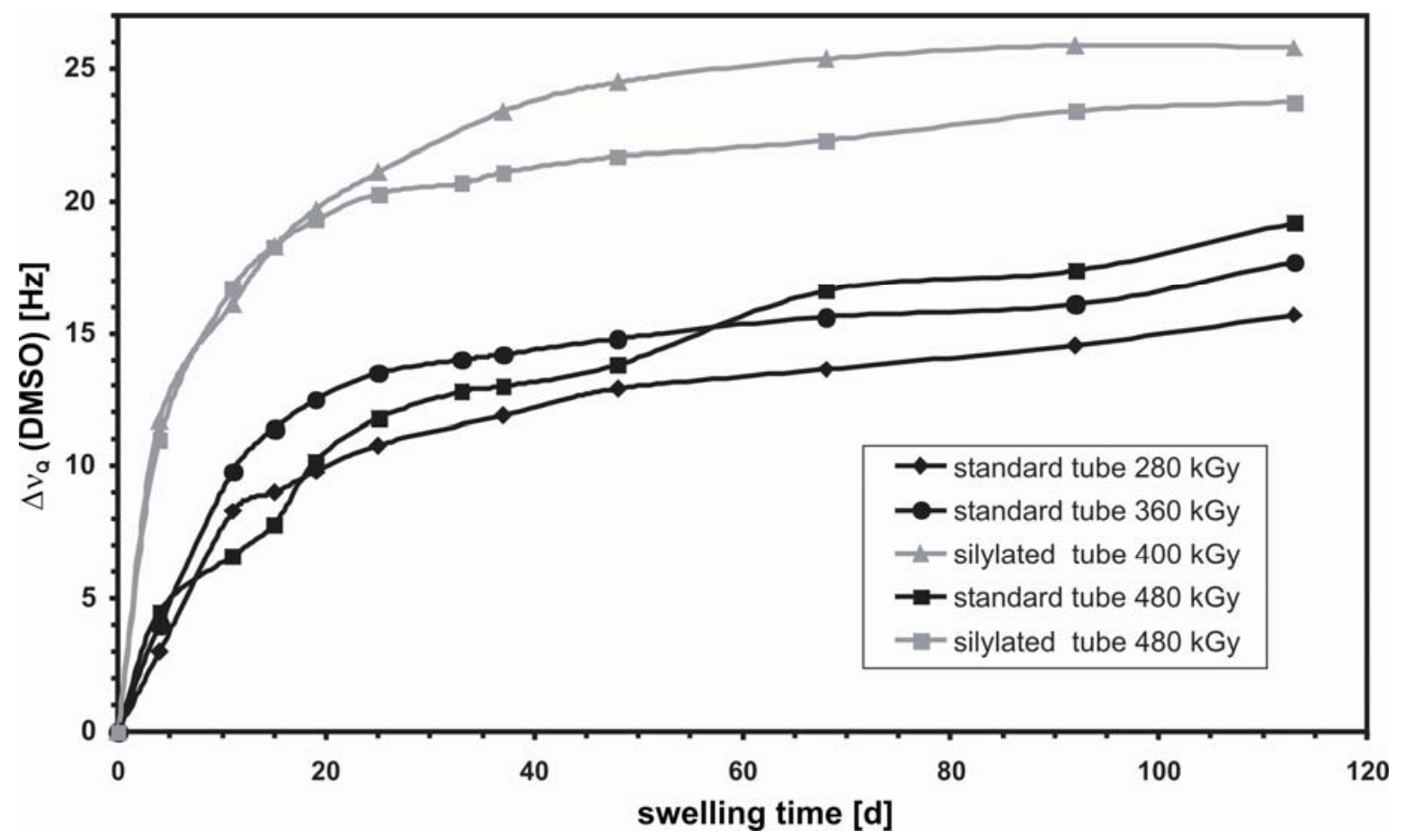

Figure S3. Quadrupolar splitting $\Delta v_{\mathrm{Q}}$ of $\mathrm{DMSO}^{-\mathrm{d}_{6}}$ taken from ${ }^{2} \mathrm{H}-1 \mathrm{D}$ spectra of five individual samples $(\mathrm{MW}=150000 \mathrm{~g} / \mathrm{mol}$ ) irradiated with $280 \mathrm{kGy}, 360 \mathrm{kGy}$, and $480 \mathrm{kGy}$ accelerated electrons in a conventional NMR-tube and $400 \mathrm{kGy}$ and $480 \mathrm{kGy}$ in NMR-tubes which were silylated prior to irradiation. Equilibration at room temperature is enhanced for silylated NMR-tubes. 



Figure S4. Suppression of polymer signals using CPMG and z-relaxation filters. (A) ${ }^{1} \mathrm{H},{ }^{13} \mathrm{C}$-HSQC without relaxation filter, (B) the same experiment with identical parameters with an additional $175 \mathrm{~ms}$ CPMG period ${ }^{1}$ applied directly after the initial excitation pulse, (C) the experiment with a z-relaxation filter of $500 \mathrm{~ms}$ incorporated as described in ${ }^{2}$. Traces show the $-\mathrm{CHCN}-$ and $-\mathrm{CH}_{2}-$ groups of the polymer and the $\mathrm{C} 3-\mathrm{H} 3 \mathrm{x}, \mathrm{C} 3-\mathrm{H} 3 \mathrm{n}$ cross peaks of $\approx 100$ mM norcamphor for comparison. All traces and contours are scaled identical. 
Table S1. Assignment and measured one-bond couplings for norcamphor in isotropic DMSO-solution and diffused into a PAN/DMSO-gel with a quadrupolar splitting $\Delta \mathrm{v}_{\mathrm{Q}}$ of $21.1 \mathrm{~Hz}$.

\begin{tabular}{|c|c|c|c|c|c|}
\hline Signal & $\begin{array}{l}\delta\left({ }^{13} \mathrm{C}\right) \\
{[\mathrm{ppm}]}\end{array}$ & $\begin{array}{c}\delta\left({ }^{1} \mathrm{H}\right) \\
{[\mathrm{ppm}]}\end{array}$ & ${ }^{1} \mathrm{~J}_{\mathrm{CH}}[\mathrm{Hz}]$ & ${ }^{1} \mathrm{~J}_{\mathrm{CH}}+\mathrm{D}_{\mathrm{CH}}[\mathrm{Hz}]$ & $\mathrm{D}_{\mathrm{CH}}[\mathrm{Hz}]$ \\
\hline $\mathrm{C} 1-\mathrm{H} 1$ & 49.0 & 2.45 & $148.8 \pm 0.2$ & $145.4 \pm 0.3$ & $-3.4 \pm 0.36$ \\
\hline $\mathrm{C} 3-\mathrm{H} 3 \mathrm{x}$ & 44.4 & 2.01 & $128.1 \pm 0.2$ & $119.9 \pm 0.5$ & $-8.2 \pm 0.54$ \\
\hline $\mathrm{C} 3-\mathrm{H} 3 \mathrm{n}$ & & 1.73 & $134.5 \pm 0.3$ & $128.6 \pm 0.5$ & $-5.9 \pm 0.58$ \\
\hline $\mathrm{C} 7-\mathrm{H} 7 \mathrm{~s}$ & 36.8 & 1.67 & $133.5 \pm 0.5$ & $127.3 \pm 0.5$ & $-6.2 \pm 0.71$ \\
\hline $\mathrm{C} 7-\mathrm{H} 7 \mathrm{a}$ & & 1.48 & $136.7 \pm 0.5$ & $143.6 \pm 0.5$ & $6.9 \pm 0.71$ \\
\hline $\mathrm{C} 4-\mathrm{H} 4$ & 36.8 & 2.57 & $144.8 \pm 0.2$ & $151.8 \pm 0.3$ & $7.0 \pm 0.36$ \\
\hline $\mathrm{C} 5-\mathrm{H} 5 \mathrm{x}$ & 34.6 & 1.71 & $131.9 \pm 0.3$ & $145.2 \pm 1.0$ & $13.3 \pm 1.04$ \\
\hline C5-H5n & & 1.48 & $133.2 \pm 0.5$ & $126.8 \pm 0.3$ & $-6.4 \pm 0.58$ \\
\hline $\mathrm{C} 6-\mathrm{H} 6 \mathrm{x}$ & 23.5 & 1.76 & $135.6 \pm 0.3$ & $130.7 \pm 1.0$ & $-4.9 \pm 1.04$ \\
\hline C6-H6n & & 1.35 & $134.1 \pm 0.3$ & $140.4 \pm 0.7$ & $6.3 \pm 0.76$ \\
\hline
\end{tabular}



Figure S5. (A) Norcamphor. (B) Alignment tensor parameters and correlation factor $\mathrm{R}^{2}$ of norcamphor as obtained by PALES $^{3}$ with the -bestFit option. (C) Experimental vs. fitted RDCs. 


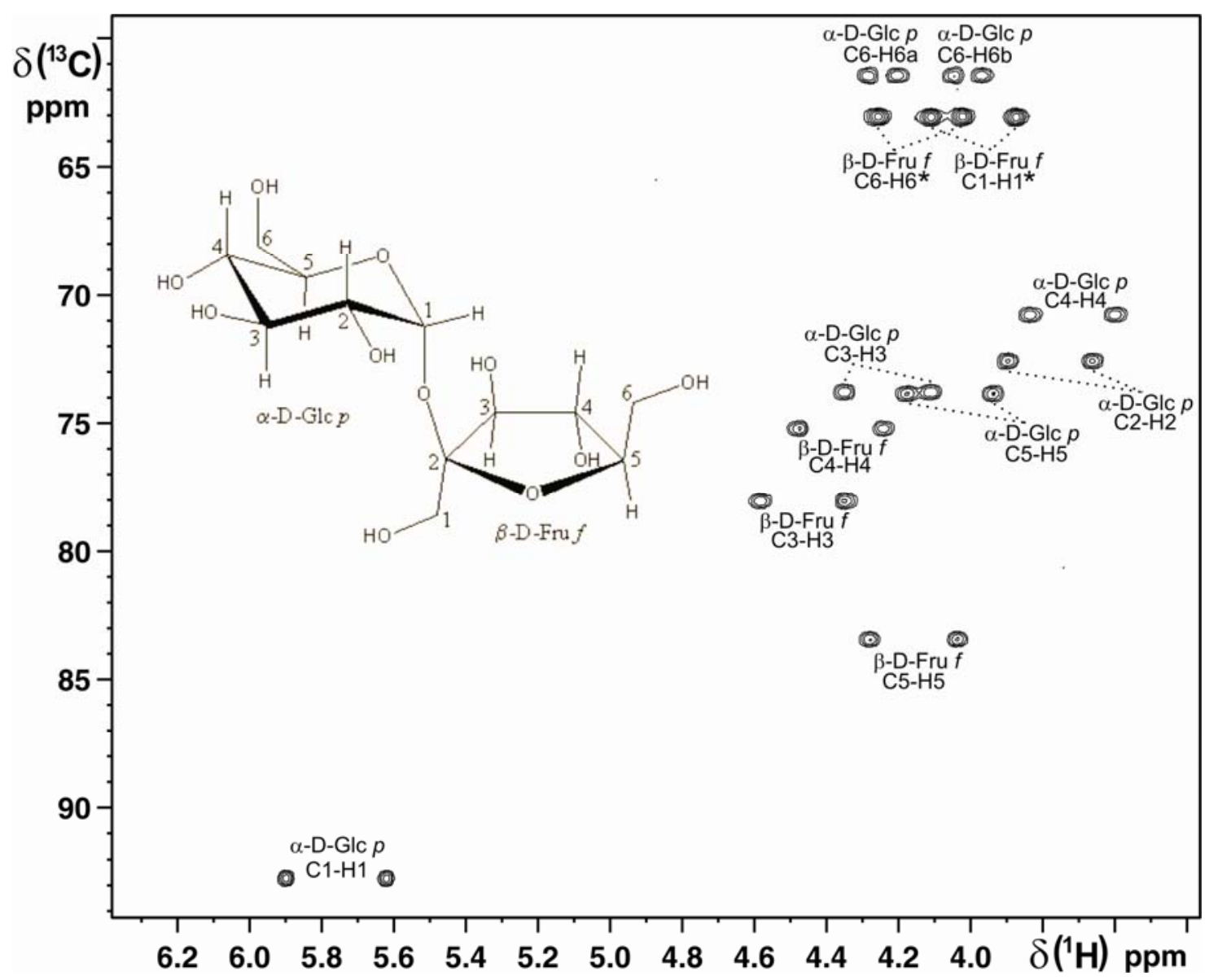

Figure S6. ${ }^{1} \mathrm{H},{ }^{13} \mathrm{C}$-HSQC of sucrose acquired inside a stretched PAN/DMSO-gel with a quadrupolar splitting $\Delta v_{\mathrm{Q}}$ of DMSO- $\mathrm{d}_{6}$ of $40.4 \mathrm{~Hz}$. The structure of sucrose with corresponding numbering of the carbon atoms is shown as inlet.

Table S2. Measured one-bond couplings of sucrose in isotropic DMSO solution and diffused into a PAN/DMSO-gel with quadrupolar DMSO- $\mathrm{d}_{6}$ deuterium splitting $\Delta \mathrm{v}_{\mathrm{Q}}$ of $40.4 \mathrm{~Hz}$.

\begin{tabular}{|c|c|c|c|c|}
\hline \multicolumn{2}{|c|}{ Signal } & ${ }^{1} \mathrm{~J}_{\mathrm{CH}}$ & ${ }^{1} \mathrm{~J}_{\mathrm{CH}}+\mathrm{D}_{\mathrm{CH}}$ & $\mathbf{D}_{\mathrm{CH}}$ \\
\hline$\alpha$-D-Glcp & $\mathrm{C} 6-\mathrm{H} 6 \mathrm{a}$ & $141.1 \pm 0.5 \mathrm{~Hz}$ & $133.3 \pm 0.8 \mathrm{~Hz}$ & $-7.8 \pm 0.9 \mathrm{~Hz}$ \\
\hline$\alpha$-D-Glcp & $\mathrm{C} 6-\mathrm{H} 6 \mathrm{~b}$ & $139.4 \pm 0.5 \mathrm{~Hz}$ & $151.5 \pm 0.8 \mathrm{~Hz}$ & $12.1 \pm 0.9 \mathrm{~Hz}$ \\
\hline$(\beta-\mathrm{D}-\mathrm{Fruf}$ & $\mathrm{C} 6-\mathrm{H} 6^{* a}$ & $140.7 \pm 1.0 \mathrm{~Hz}$ & $135.5 \pm 0.6 \mathrm{~Hz}$ & $-5.2 \pm 1.2 \mathrm{~Hz}$ \\
\hline$(\beta-D-F r u f$ & $\mathrm{C} 1-\mathrm{H} 1^{* a}$ & $141.9 \pm 0.3 \mathrm{~Hz}$ & $144.9 \pm 0.6 \mathrm{~Hz}$ & $3.0 \pm 0.7 \mathrm{~Hz}$ \\
\hline$\alpha$-D-Glcp & $\mathrm{C} 4-\mathrm{H} 4$ & $141.7 \pm 0.4 \mathrm{~Hz}$ & $146.1 \pm 0.5 \mathrm{~Hz}$ & $4.4 \pm 0.6 \mathrm{~Hz}$ \\
\hline$\alpha$-D-Glcp & $\mathrm{C} 2-\mathrm{H} 2$ & $141.1 \pm 0.6 \mathrm{~Hz}$ & $148.2 \pm 0.3 \mathrm{~Hz}$ & $7.1 \pm 0.7 \mathrm{~Hz}$ \\
\hline$\alpha$-D-GIcp & $\mathrm{C} 3-\mathrm{H} 3$ & $143.5 \pm 0.7 \mathrm{~Hz}$ & $150.0 \pm 0.8 \mathrm{~Hz}$ & $6.5 \pm 1.1 \mathrm{~Hz}$ \\
\hline$\alpha$-D-Glcp & $\mathrm{C} 5-\mathrm{H} 5$ & $143.3 \pm 0.7 \mathrm{~Hz}$ & $150.0 \pm 0.8 \mathrm{~Hz}$ & $6.7 \pm 1.1 \mathrm{~Hz}$ \\
\hline$\beta$-D-Fruf & $\mathrm{C} 4-\mathrm{H} 4$ & $144.2 \pm 1.0 \mathrm{~Hz}$ & $128.8 \pm 0.5 \mathrm{~Hz}$ & $-15.4 \pm 1.1 \mathrm{~Hz}$ \\
\hline$\beta$-D-Fruf & $\mathrm{C} 3-\mathrm{H} 3$ & $144.9 \pm 1.0 \mathrm{~Hz}$ & $126.2 \pm 0.5 \mathrm{~Hz}$ & $-18.7 \pm 1.1 \mathrm{~Hz}$ \\
\hline$\beta$-D-Fruf & $\mathrm{C} 5-\mathrm{H} 5$ & $146.2 \pm 0.6 \mathrm{~Hz}$ & $128.4 \pm 0.5 \mathrm{~Hz}$ & $-17.8 \pm 0.8 \mathrm{~Hz}$ \\
\hline$\alpha$-D-GIcp & $\mathrm{C} 1-\mathrm{H} 1$ & $167.2 \pm 0.4 \mathrm{~Hz}$ & $182.5 \pm 0.4 \mathrm{~Hz}$ & $15.3 \pm 0.6 \mathrm{~Hz}$ \\
\hline
\end{tabular}

${ }^{a}$ individual protons have not been resolved and measured RDCs can not be used for structural input 
A

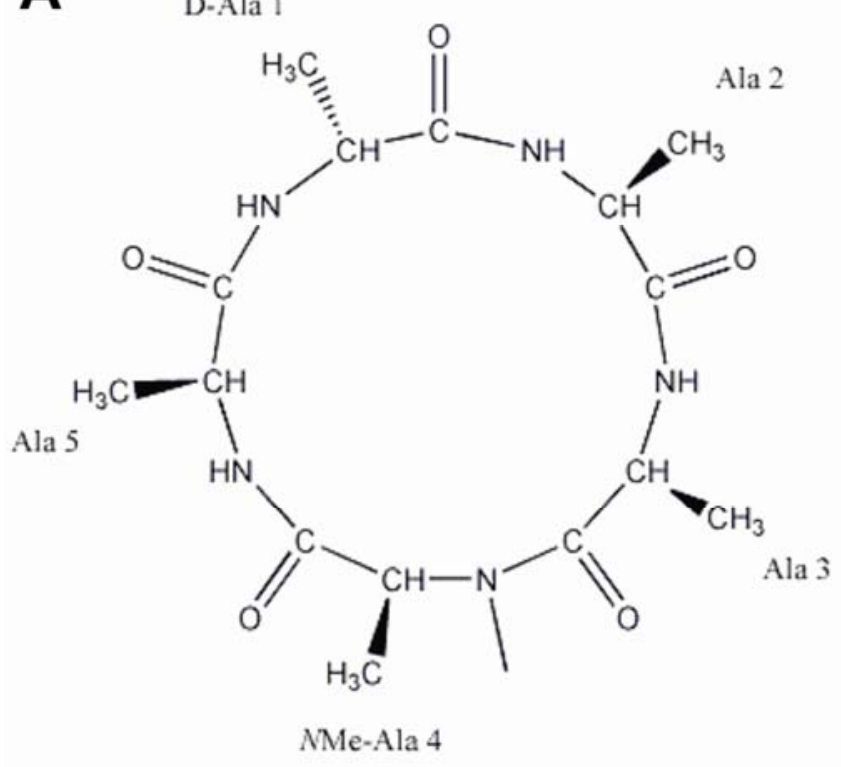

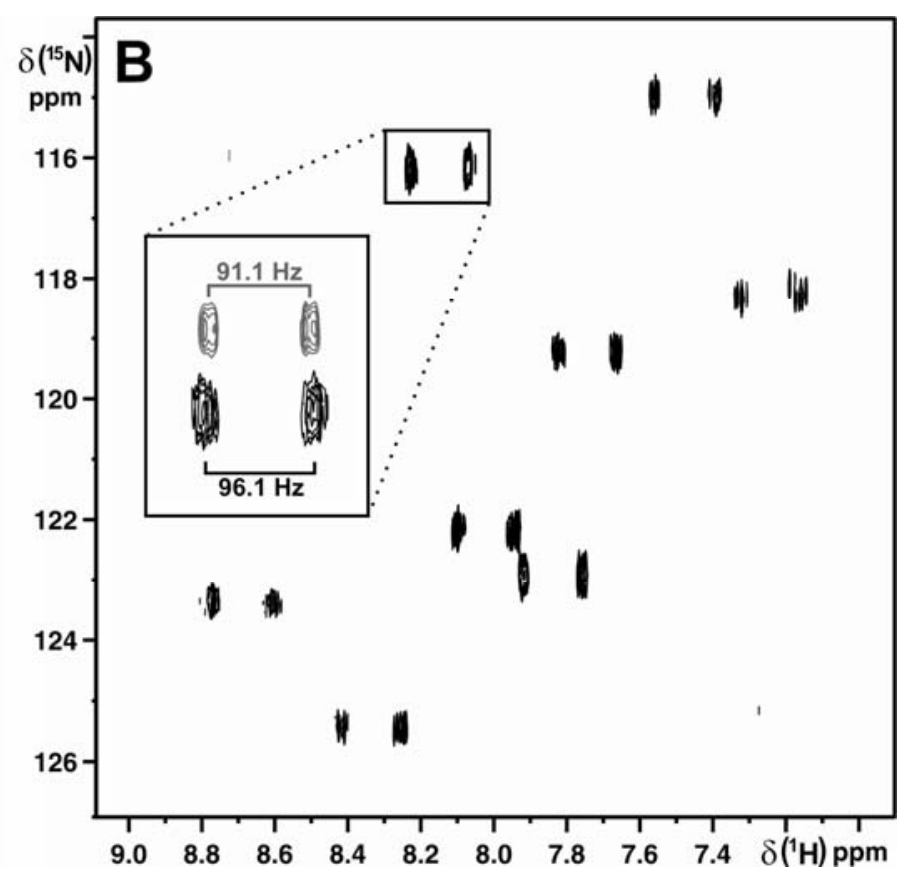

Figure S7. Structure (A) and coupled ${ }^{1} \mathrm{H},{ }^{15} \mathrm{~N}-\mathrm{HSQC}(\mathrm{B})$ of the cyclic pentapeptide cyclo(-D-Ala-Ala-Ala-(NMe)Ala-Ala-) inside the PAN/DMSO-gel with $\Delta v_{\mathrm{Q}}=21.2 \mathrm{~Hz}$. The inlet compares the amide group of Ala 3 of conformer 1 with the cross peak in isotropic DMSO-d ${ }_{6}$-solution (in gray).

Table S3. ${ }^{1} \mathrm{H},{ }^{15} \mathrm{~N}$ and ${ }^{13} \mathrm{C}$ assignment for the two conformations of cyclo(-D-Ala-Ala-Ala-(NMe)Ala-Ala-). Isotropic chemical shift values are given in ppm.

\begin{tabular}{|l|c|c|c|c|c|c|c|c|}
\hline & $\mathbf{H} \boldsymbol{\alpha}$ & $\mathbf{H} \boldsymbol{\beta}$ & $\mathbf{H}_{\boldsymbol{N}-\mathbf{M e}}$ & $\mathbf{H N}$ & $\mathbf{N}$ & $\mathbf{C} \boldsymbol{C}$ & $\mathbf{C} \boldsymbol{\beta}$ & $\mathbf{C O}$ \\
\hline conformer 1 & & & & & & & & \\
\hline D-Ala 1 & 4.33 & 1.13 & - & 8.05 & 122.2 & 47.0 & 16.2 & 170.4 \\
\hline Ala 2 & 4.26 & 1.19 & - & 7.23 & 118.3 & 50.4 & 18.9 & 171.0 \\
\hline Ala 3 & 4.41 & 1.19 & - & 8.15 & 116.2 & 45.2 & 16.7 & 169.9 \\
\hline NMe-Ala 4 & 4.48 & 1.29 & 2.59 & - & - & 55.4 & 14.1 & 168.5 \\
\hline Ala 5 & 4.37 & 1.18 & - & 7.81 & 122.9 & 47.1 & 16.5 & 170.1 \\
\hline & & & & & & & & \\
\hline conformer 2 & & & & & & & & \\
\hline D-Ala 1 & 4.10 & 1.16 & - & 8.38 & 125.5 & 49.2 & 16.4 & 172.5 \\
\hline Ala 2 & 3.93 & 1.27 & - & 8.68 & 123.4 & 49.6 & 16.6 & 171.1 \\
\hline Ala 3 & 4.61 & 1.09 & - & 7.48 & 115.0 & 45.1 & 17.7 & 171.5 \\
\hline NMe-Ala 4 & 3.52 & 1.54 & 2.85 & - & - & 64.6 & 14.8 & 171.6 \\
\hline Ala 5 & 4.45 & 1.17 & - & 7.74 & 119.1 & 47.1 & 16.0 & 169.9 \\
\hline
\end{tabular}


Table S4. One-bond couplings measured for conformer 1 of the pentapeptide cyclo(-D-Ala-Ala-Ala-(NMe)Ala-Ala-) in isotropic DMSO- $\mathrm{d}_{6}$ solution and inside the PAN/DMSO- $\mathrm{d}_{6}$ gel with $\Delta \mathrm{v}_{\mathrm{Q}}=21.2 \mathrm{~Hz}$.

\begin{tabular}{|c|c|c|c|c|}
\hline \multicolumn{2}{|c|}{ Signal } & ${ }^{1} \mathrm{~J}_{\mathrm{XH}}[\mathrm{Hz}]$ & ${ }^{1} J_{X H}+D_{X H}[H z]$ & $\mathrm{D}_{\mathrm{XH}}[\mathrm{Hz}]$ \\
\hline D-Ala 1 & $\mathrm{C} \beta-\mathrm{H} \beta$ & $128.3 \pm 0.1$ & $128.7 \pm 0.3$ & $0.4 \pm 0.3$ \\
\hline D-Ala 1 & $\mathrm{C} \alpha-\mathrm{H} \alpha$ & $136.6 \pm 0.3$ & $142.3 \pm 2.0$ & $5.7 \pm 2.0$ \\
\hline D-Ala 1 & $\mathrm{~N}-\mathrm{NH}$ & $91.2 \pm 0.3$ & $94.6 \pm 2.5$ & $3.4 \pm 2.5$ \\
\hline Ala 2 & $\mathrm{C} \beta-\mathrm{H} \beta$ & $128.5 \pm 0.1$ & $127.8 \pm 0.5$ & $-0.8 \pm 0.5$ \\
\hline Ala 2 & $\mathrm{C} \alpha-\mathrm{H} \alpha$ & $143.3 \pm 0.2$ & $157.8 \pm 2.5$ & $14.5 \pm 2.5$ \\
\hline Ala 2 & $\mathrm{~N}-\mathrm{NH}$ & $91.0 \pm 0.7$ & $95.8 \pm 1.5$ & $4.8 \pm 1.7$ \\
\hline Ala 3 & $\mathrm{C} \beta-\mathrm{H} \beta$ & $128.7 \pm 0.2$ & $130.7 \pm 0.6$ & $2.0 \pm 0.6$ \\
\hline Ala 3 & $\mathrm{C} \alpha-\mathrm{H} \alpha$ & $138.2 \pm 0.3$ & $150.6 \pm 2.0$ & $12.4 \pm 2.0$ \\
\hline Ala 3 & $\mathrm{~N}-\mathrm{NH}$ & $91.1 \pm 0.2$ & $96.1 \pm 1.5$ & $5.0 \pm 1.5$ \\
\hline NMe-Ala 4 & $\mathrm{C} \beta-\mathrm{H} \beta$ & $128.5 \pm 0.2$ & $130.3 \pm 0.6$ & $1.8 \pm 0.6$ \\
\hline NMe-Ala 4 & $\mathrm{C} \alpha-\mathrm{H} \alpha$ & $134.9 \pm 0.4$ & $142.3 \pm 2.0$ & $7.4 \pm 2.0$ \\
\hline NMe-Ala 4 & $\mathrm{Cm}-\mathrm{Hm}$ & $138.8 \pm 0.1$ & $136.0 \pm 0.8$ & $-2.8 \pm 0.8$ \\
\hline Ala 5 & $\mathrm{C} \beta-\mathrm{H} \beta$ & $128.1 \pm 0.1$ & $123.6 \pm 0.5$ & $-4.5 \pm 0.5$ \\
\hline Ala 5 & $\mathrm{C} \alpha-\mathrm{H} \alpha$ & $136.5 \pm 0.5$ & $142.5 \pm 3.5$ & $6.0 \pm 3.5$ \\
\hline Ala 5 & $\mathrm{~N}-\mathrm{NH}$ & $91.5 \pm 0.2$ & $95.1 \pm 1.5$ & $3.6 \pm 1.5$ \\
\hline
\end{tabular}

Table S5. One-bond couplings measured for conformer 2 of the pentapeptide cyclo(-D-Ala-Ala-Ala-(NMe)Ala-Ala-) in isotropic DMSO- $\mathrm{d}_{6}$ solution and inside the PAN/DMSO- $\mathrm{d}_{6}$ gel with $\Delta \mathrm{v}_{\mathrm{Q}}=21.2 \mathrm{~Hz}$.

\begin{tabular}{|ll|r|r|r|r|}
\hline \multicolumn{2}{|c|}{ Signal } & \multicolumn{2}{|c|}{${ }^{1} \mathbf{J}_{\mathbf{X H}}[\mathrm{Hz}]$} & \multicolumn{1}{c|}{$\mathbf{1}_{\mathbf{X H}}+\mathbf{D}_{\mathbf{X H}}[\mathrm{Hz}]$} & $\mathbf{D}_{\mathbf{X H}}[\mathrm{Hz}]$ \\
\hline D-Ala 1 & $\mathrm{C} \beta-\mathrm{H} \beta$ & $128.3 \pm 0.1$ & $122.8 \pm 2.0$ & $-5.5 \pm 2.0$ \\
\hline D-Ala 1 & $\mathrm{C} \alpha-\mathrm{H} \alpha$ & $142.8 \pm 0.4$ & $165.6 \pm 1.0$ & $22.8 \pm 1.1$ \\
\hline D-Ala 1 & $\mathrm{N}-\mathrm{NH}$ & $92.8 \pm 0.3$ & $98.2 \pm 1.5$ & $5.4 \pm 1.5$ \\
\hline Ala 2 & $\mathrm{C} \beta-\mathrm{H} \beta$ & $129.1 \pm 0.1$ & $136.4 \pm 1.3$ & $7.3 \pm 1.3$ \\
\hline Ala 2 & $\mathrm{C} \alpha-\mathrm{H} \alpha$ & $143.5 \pm 0.3$ & $171.3 \pm 3.0$ & $27.8 \pm 3.0$ \\
\hline Ala 2 & $\mathrm{N}-\mathrm{NH}$ & $92.4 \pm 8.0$ & $104.5 \pm 5.0$ & $12.1 \pm 9.4$ \\
\hline Ala 3 & $\mathrm{C} \beta-\mathrm{H} \beta$ & $128.5 \pm 0.2$ & $128.4 \pm 0.6$ & $-0.1 \pm 0.6$ \\
\hline Ala 3 & $\mathrm{C} \alpha-\mathrm{H} \alpha$ & $139.3 \pm 0.3$ & $175.7 \pm 1.5$ & $36.4 \pm 1.5$ \\
\hline Ala 3 & $\mathrm{N}-\mathrm{NH}$ & $91.6 \pm 0.3$ & $102.2 \pm 5.0$ & $10.6 \pm 5.0$ \\
\hline NMe-Ala 4 & $\mathrm{C} \beta-\mathrm{H} \beta$ & $129.0 \pm 0.1$ & $123.6 \pm 0.5$ & $-5.4 \pm 0.5$ \\
\hline NMe-Ala 4 & $\mathrm{C} \alpha-\mathrm{H} \alpha$ & $139.8 \pm 0.8$ & $159.3 \pm 4.0$ & $19.5 \pm 4.1$ \\
\hline NMe-Ala 4 & $\mathrm{Cm}-\mathrm{Hm}$ & $138.8 \pm 0.1$ & $131.3 \pm 1.0$ & $-7.5 \pm 1.0$ \\
\hline Ala 5 & $\mathrm{C} \beta-\mathrm{H} \beta$ & $128.7 \pm 0.1$ & $130.3 \pm 1.0$ & $1.6 \pm 1.0$ \\
\hline Ala 5 & $\mathrm{C} \alpha-\mathrm{H} \alpha$ & $141.4 \pm 0.5$ & $158.4 \pm 5.0$ & $17.0 \pm 5.0$ \\
\hline Ala 5 & $\mathrm{N}-\mathrm{NH}$ & $89.7 \pm 0.5$ & $94.04 \pm 3.0$ & $4.3 \pm 3.0$ \\
\hline
\end{tabular}




\section{Gel preparation:}

\section{Non-cross-linked polymers:}

Two kinds of linear poly(acrylonitrile) were used:

1. Poly(acrylonitrile) purchased by Polyscience, Inc. (MW=150000 g/mol)

2. Poly(acrylonitrile) polymerized in-house by precipitation polymerization in water with the redox system $\mathrm{K}_{2} \mathrm{~S}_{2} \mathrm{O}_{8} / \mathrm{K}_{2} \mathrm{~S}_{2} \mathrm{O}_{5}$ as radical initiator $\left(\mathrm{M}_{\mathrm{n}, \mathrm{osm}}=231000 \mathrm{~g} / \mathrm{mol}\right)$.

\section{Precipitation of PAN-sticks:}

Sticks of poly(acrylonitrile) were obtained by precipitation out of a dimethylsulfoxide solution: A highly viscous solution of PAN in DMSO $(\approx 300 \mathrm{~g} / \mathrm{L})$ was filled in paper rolls with inner diameters of $3.5-4.5 \mathrm{~mm}$. The filled paper rolls were then placed in solutions with stepwise increased content of methanol: 1 . DMSO/MeOH 2:1 $\left(20 \mathrm{~min}, 80^{\circ} \mathrm{C}\right)$, 2. DMSO/MeOH 1:1 $\left(20 \mathrm{~min}, 65^{\circ} \mathrm{C}\right)$, 3. pure $\mathrm{MeOH}\left(1 \mathrm{~h}, 50^{\circ} \mathrm{C}\right)$. Finally, the paper was removed and precipitated PAN-sticks were dried at $70^{\circ} \mathrm{C}$ for $24 \mathrm{~h}$. By this procedure PAN-sticks of 2.5-3.5 mm were obtained.

\section{$\underline{\text { PAN/DMSO- }}{ }_{6}$-solutions for irradiation:}

PAN/DMSO-d - $_{6}$-solutions with concentrations of $140 \mathrm{~g} / \mathrm{L}, 210 \mathrm{~g} / \mathrm{L}$ and $280 \mathrm{~g} / \mathrm{L}$ were filled in standard $5 \mathrm{~mm}-\mathrm{NMR}$-tubes and in silylated NMR-tubes. Tubes were silylated by treatment with a 1:1 mixture of $\mathrm{ClSi}\left(\mathrm{CH}_{3}\right)_{3}$ and $\mathrm{Cl}_{2} \mathrm{Si}\left(\mathrm{CH}_{3}\right)_{2}$ for $24 \mathrm{~h}$ at room temperature and washed 3 times with dichloromethane before drying.

\section{Cross-linking by irradiation:}

PAN-sticks and PAN/DMSO- $\mathrm{d}_{6}$-solutions were irradiated with $10 \mathrm{MeV}$ electrons generated by an industrial electron accelerator (Beta-Gamma-Systems in Saal an der Donau, Germany). Sticks and tubes were placed perpendicular to the electron beam during irradiation.

The samples were irradiated by single irradiation doses of $40 \mathrm{kGy}$ which took several seconds each. The time interval between two subsequent irradiation steps was approximately 10-15 min. Total irradiation doses of $120-480$ kGy were applied.

\section{References}

(1) Carr, H. Y.; Purcell, E. M. Phys. Rev. 1954, 94, 630.

(2) Luy, B.; Kobzar, K.; Knör, S.; Furrer, J.; Heckmann, D.; Kessler, H. J. Am. Chem. Soc. 2005, 127, 6459-6465.

(3) Zweckstetter, M.; Bax, A. J. Am. Chem. Soc. 2000, 122, 3791-3792. 